\title{
NUMERICAL AND THEORETICAL STUDY ON PARAMETER SELECTION OF ACOUSTIC LOAD TEST OF STEAM DRYER
}

\author{
Jia Zhong \\ State Nuclear Power Technology R\&D Center, \\ SPICRI \\ South Area, Future Science and Technology Park, \\ Bei Jing, China, 102209
}

\author{
Han Wang \\ State Nuclear Power Technology R\&D Center, \\ SPICRI \\ South Area, Future Science and Technology Park, \\ Bei Jing, China, 102209
}

\author{
Nan Wang \\ State Nuclear Power Technology \\ R\&D Center, SPICRI \\ South Area, Future Science and \\ Technology Park, \\ Bei Jing, China, 102209
}

\author{
Jinyi Zhang \\ State Nuclear Power Technology \\ R\&D Center, SPICRI \\ South Area, Future Science and \\ Technology Park, \\ Bei Jing, China, 102209
}

\author{
Yujia Liu \\ State Nuclear Power Technology \\ R\&D Center, SPICRI \\ South Area, Future Science and \\ Technology Park, \\ Bei Jing, China, 102209
}

Wen Liu

Institute of Mechanics, Chinese Academy of Sciences

North Fourth Ring Road, NO.15

Bei Jing, China, 100190

\section{Kai Zhang}

Shanghai Nuclear Engineering Research and Design Institute of SNPTC NO.29 Hongcao Road, Shang Hai, China, 200233

\begin{abstract}
In recent years some reactors have experienced significant steam dryer cracking. The cause of the dryer's failure was considered as flow-induced acoustic resonance at the stub pipes of the safety relief valves (SRVs) on the main steam lines (MSLs). A research program was started to investigate the acoustic resonance of the steam dryer. Most studies used scaled down method, while our research based on the full scale steam dryer of the prototype CAP1400 but with only one unit of the steam dryer. The purpose of our research program is to study the strength of the steam dryer under flow-induced acoustic resonance. This paper introduces the parameter selection of the main pipe and the stub pipe through numerical method and theoretical method.
\end{abstract}

\section{INTRODUCTION}

In recent years some reactors have experienced significant steam dryer cracking. The detailed inspection of the stem dryer showed that it was likely that the steam dryer had been damaged by high cycle fatigue due to acoustic-induced vibration[1,2]. The cause of the dryer's failure was considered as flow-induced acoustic resonance at the stub pipes of the safety relief valves (SRVs) on the main steam lines (MSLs). Acoustic resonance was considered to be generated by the interaction between the sound field and an unstable shear layer across the closed side branches of the SRV stub pipes (shown in Fig. 1), where the fluctuating pressure or sound was generated by unsteady motion of a vortex. And the resonance of the quarter wavelength mode is excited by the vortex sound in the SRV stub pipe. If the shedding frequency of the vortex is close to the resonance frequency, the shedding frequency of the vortex will be locked at the resonance frequency. Through this feedback mechanism, a highly intense fluctuating pressure occurs in the SRV stub pipe[3]. 


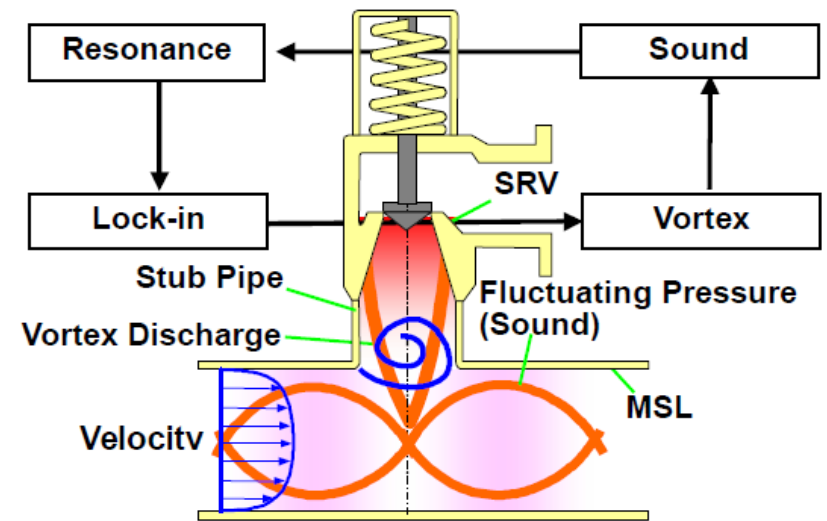

Fig. 1 Flow-induced acoustic resonance at the SRV stub pipe

The phenomenon of flow-induced acoustic resonance has been investigated by many researchers[3-10]. Most studies used scaled down method, while our research based on the full scale steam dryer of the prototype CAP1400 but with only one unit of the steam dryer. The purpose of our research program is to study the strength of the steam dryer under flow-induced acoustic resonance. The schematic diagram of the test facility is shown in Fig. 2. The test facility consists of a compressor, a gasholder, a test vessel, a steam dryer, a main pipe, a stub pipe, a stable section, a throatable nozzle, a super expansion section and a silencer. In order to get raise the sound power level (SPL) of the steam dryer to the highest, theoretically analysis was used to confirm the parameters of the test facility. This paper will introduce the parameter selection of the main pipe and the stub pipe through numerical method and theoretical method.

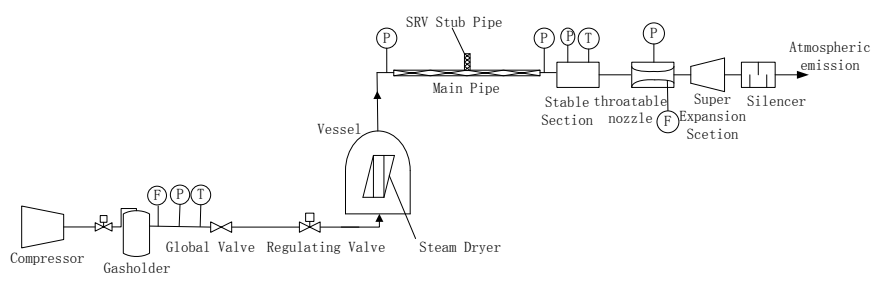

Fig. 2 Schematic diagram of the test facility

\section{NUMERICAL ANALYSIS}

Based on ANSYS, we study the effect of the aerodynamic noise on four parameters which are shown in Fig. 3 (the height of the stub pipe $\mathrm{H}$, the velocity of the main pipe $\mathrm{V}$, the length of the main pipe $\mathrm{L}$ and the diameter of the main pipe D). Figure 4 shows the position of the measuring points.

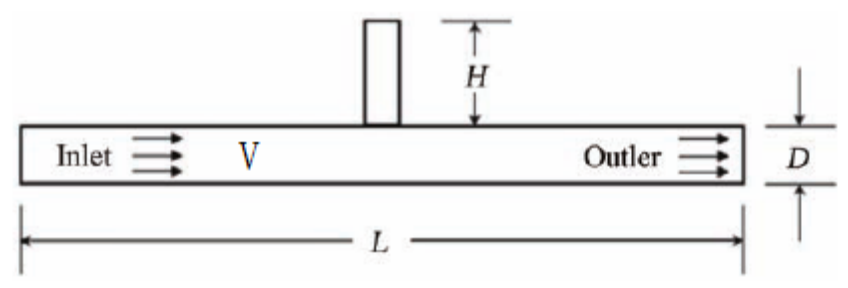

Fig. 3 Schematic of the simplified acoustic source model

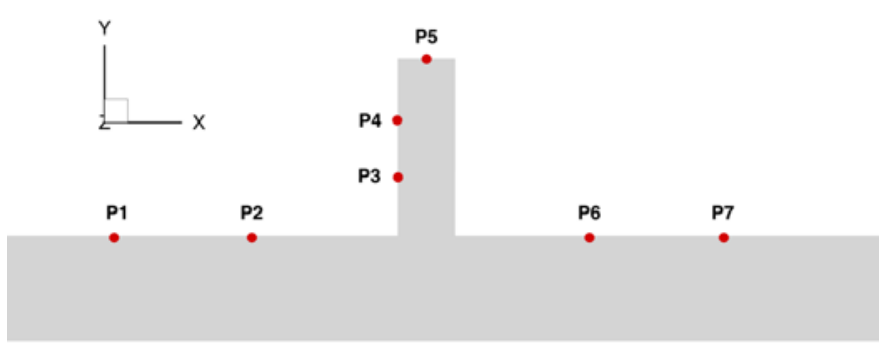

Fig. 4 Schematic of the position of the measuring point

\subsection{THE INFLUENCE OF THE HEIGHT OF THE STUB PIPE}

In this case, three other parameters (the length of the main pipe, the diameter of the main pipe, and the velocity of the main pipe) were fixed and the height of the stub pipe was changed to investigate its influence on SPL. The working conditions of the compared numerical examples are shown in Table 1.

Table 1 Working conditions 2.1

\begin{tabular}{c|c|c|c|c}
\hline Working condition & $\begin{array}{c}\mathbf{H} \\
\mathbf{( m m})\end{array}$ & $\begin{array}{c}\mathrm{L} \\
(\mathrm{mm})\end{array}$ & $\begin{array}{c}\mathrm{D} \\
(\mathrm{mm})\end{array}$ & $\begin{array}{c}\text { V } \\
(\mathrm{m} / \mathrm{s})\end{array}$ \\
\hline I & $\mathbf{2 4 0}$ & 3000 & 110 & 45 \\
II & $\mathbf{1 8 5}$ & 3000 & 110 & 45 \\
III & $\mathbf{6 5}$ & 3000 & 110 & 45 \\
\hline
\end{tabular}

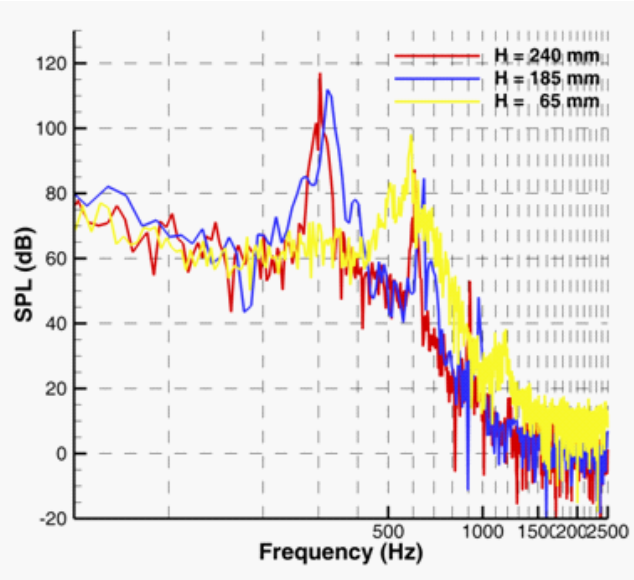

a)

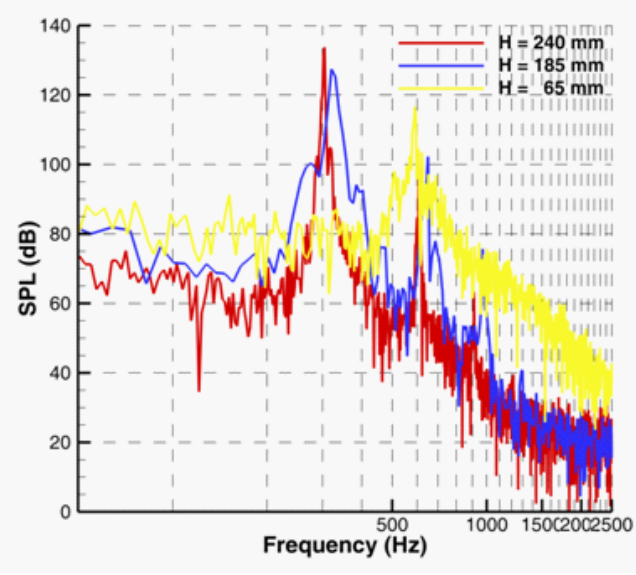

b)

Fig. 5 Sound pressure frequency spectrum curve: a) p2; b) p5 
The curve of SPL with frequency is shown in Fig. 5. At different measuring points, there is a strong monotonic noise. When the height of the stub pipe is much greater than the diameter of the stub pipe, the main peak frequency is about $310 \sim 330 \mathrm{~Hz}$ which is similar to the first-order modality of the stub pipe. When the height of the stub pipe is close to the diameter of the stub pipe, the main peak frequency is close to the second-order modality of the stub pipe.

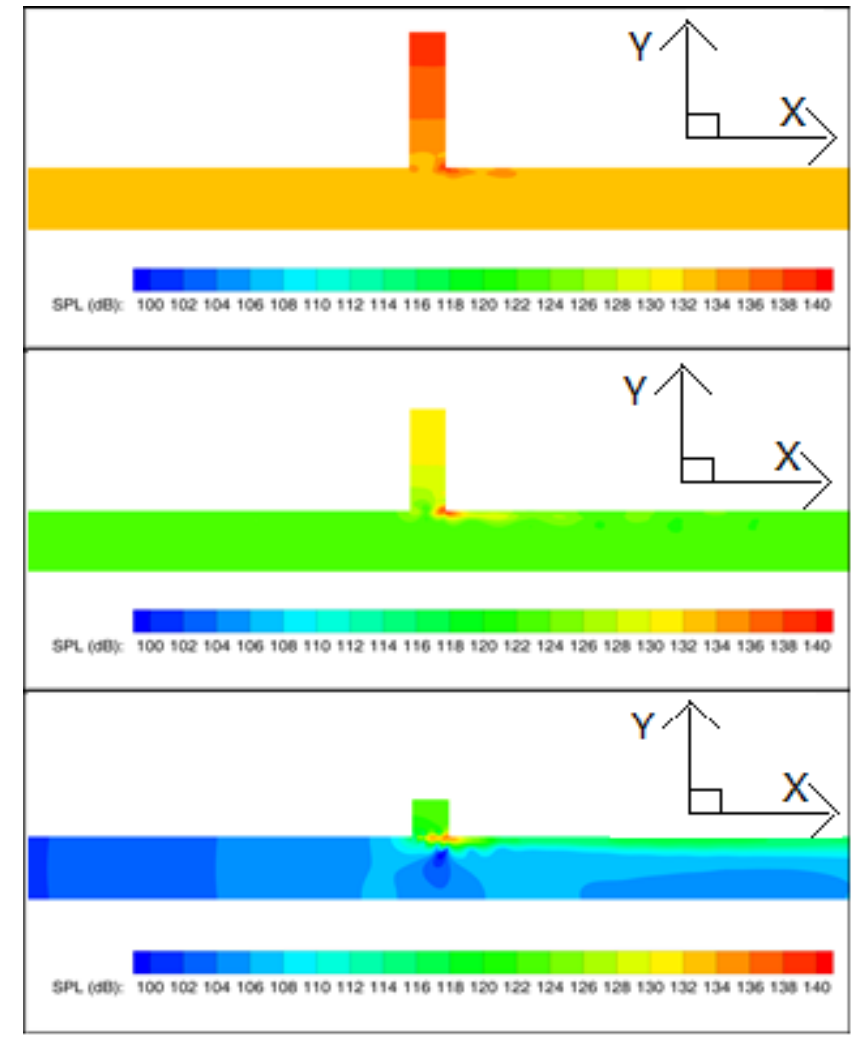

Fig. 6 SPL distribution map under different height of the stub pipe

Figure 6 shows the distribution of SPL in the pipe under different height of the stub pipe. It can be seen that SPL increases as the height of stub pipe increases.

\subsection{THE INFLUENCE OF THE LENGTH OF THE MAIN PIPE}

In this case, three other parameters (the height of the stub pipe, the diameter of the main pipe and the velocity of the main pipe) were fixed and the length of the main pipe was changed to investigate its influence on SPL. The working conditions of the compared numerical example are shown in Table 2.

Table 2 Working conditions 2.2

\begin{tabular}{c|c|c|c|c}
\hline Working condition & $\begin{array}{c}\text { H } \\
(\mathrm{mm})\end{array}$ & $\begin{array}{c}\mathbf{L} \\
(\mathbf{m m})\end{array}$ & $\begin{array}{c}\mathrm{D} \\
(\mathrm{mm})\end{array}$ & $\begin{array}{c}\text { V } \\
(\mathrm{m} / \mathrm{s})\end{array}$ \\
\hline I & 240 & $\mathbf{3 0 0 0}$ & 110 & 45 \\
II & 240 & $\mathbf{2 3 0 0}$ & 110 & 45 \\
\hline
\end{tabular}

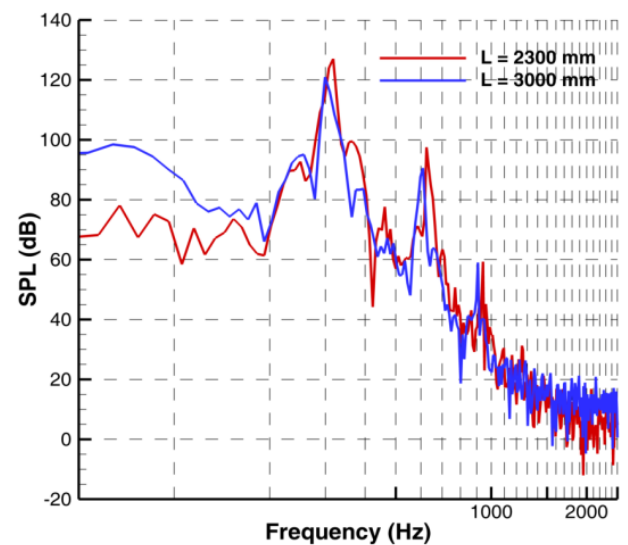

a)

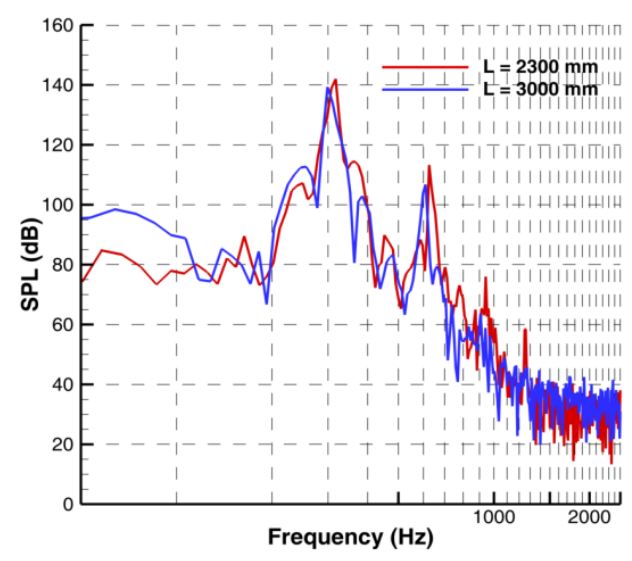

b)

Fig. 7 Sound pressure frequency spectrum curve: a) p2; b) p5

In Fig.7 it can be seen that with the increase of the length of the main pipe, the peak frequency is almost the same, but the strength slightly decreases. Taking the calculation of the grid as well as the discrete error into account, the effect of the length changes of the main pipe on SPL can be ignored.

\subsection{THE INFLUENCE OF THE DIAMETER OF THE MAIN PIPE}

In this case, three other parameters (the height of the stub pipe, the length of the main pipe and the velocity of the main pipe) were fixed and the diameter of the main pipe was changed to investigate its influence on SPL. The working conditions of the compared numerical example are shown in Table 3.

Table 3 Working conditions 2.3

\begin{tabular}{c|c|c|c|c}
\hline Working condition & $\begin{array}{c}\mathrm{H} \\
(\mathrm{mm})\end{array}$ & $\begin{array}{c}\mathrm{L} \\
(\mathrm{mm})\end{array}$ & $\begin{array}{c}\mathbf{D} \\
\mathbf{( m m})\end{array}$ & $\begin{array}{c}\mathrm{V} \\
(\mathrm{m} / \mathrm{s})\end{array}$ \\
\hline I & 240 & 2300 & $\mathbf{1 1 0}$ & 45 \\
II & 240 & 2300 & $\mathbf{2 2 0}$ & 45 \\
\hline
\end{tabular}

In Fig. 8 it can be seen that the change of the diameter of the main pipe has a significant influence on the flow field in 
the pipe. With the increase of the diameter of the main pipe, the $\mathrm{Y}$ direction of spatial scales increase which makes the flow more intensely.

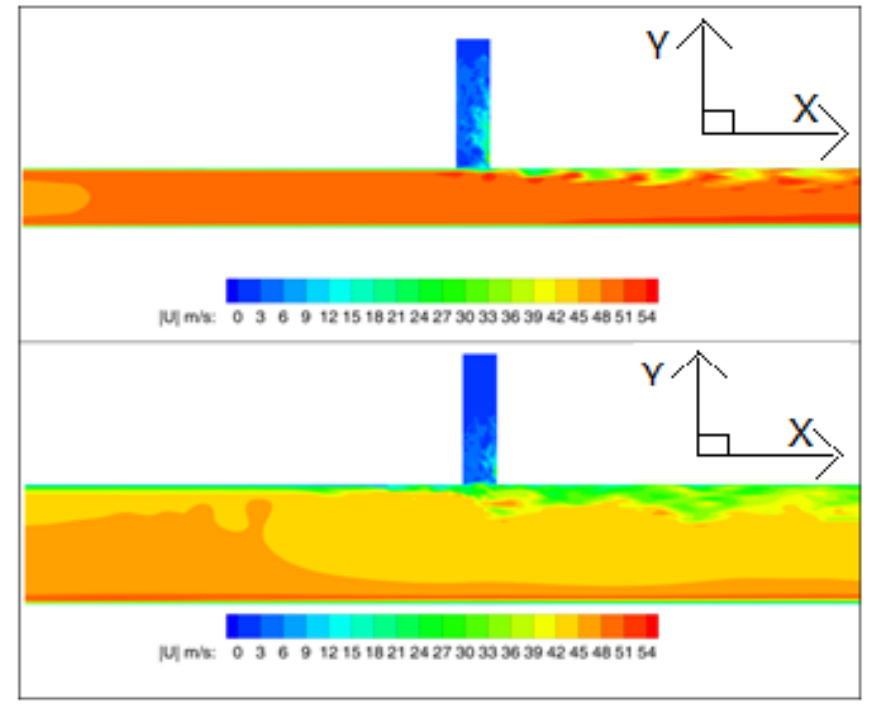

Fig. 8 Instantaneous velocity field under different diameter of the main pipe

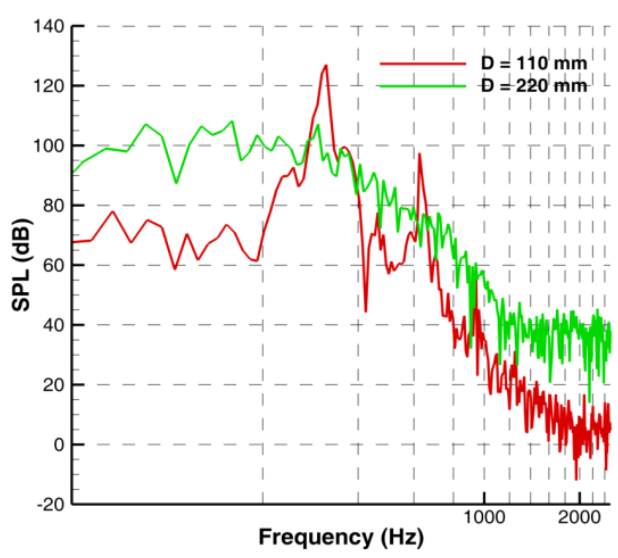

a)

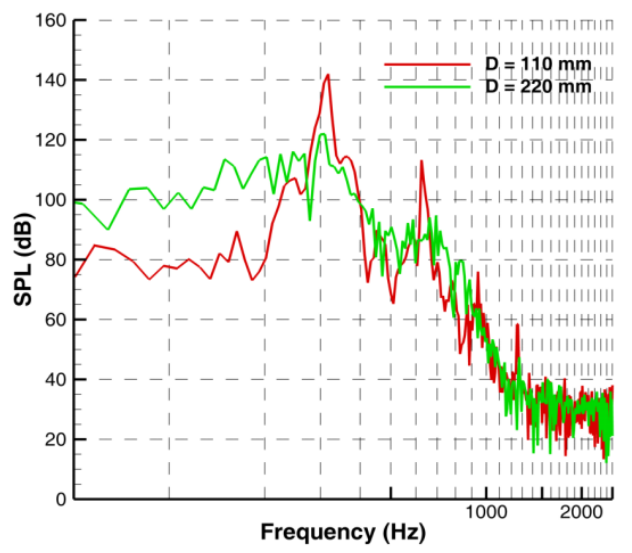

b)

Fig. 9 Sound pressure frequency spectrum curve: a) p2; b) p5
In Fig. 9 it can be found that the noise with the $110 \mathrm{~mm}$ diameter appears obvious monotonic peak frequencies, while the noise of $220 \mathrm{~mm}$ diameter is more like a broadband noise.

\subsection{THE INFLUENCE OF THE VELOCITY OF THE MAIN PIPE}

In this case, three other parameters (the diameter of the main pipe, the length of the main pipe and the height of the stub pipe) were fixed and the velocity of the main pipe was changed to investigate its influence on SPL. The working conditions of the compared numerical example are shown in Table 4.

Table 4 Working conditions 2.4

\begin{tabular}{c|c|c|c|c}
\hline Working condition & $\begin{array}{c}\mathrm{H} \\
(\mathrm{mm})\end{array}$ & $\begin{array}{c}\mathrm{L} \\
(\mathrm{mm})\end{array}$ & $\begin{array}{c}\mathrm{D} \\
(\mathrm{mm})\end{array}$ & $\begin{array}{c}\mathbf{V} \\
\mathbf{( m / s})\end{array}$ \\
\hline I & 185 & 3000 & 110 & $\mathbf{3 0}$ \\
II & 185 & 3000 & 110 & $\mathbf{4 5}$ \\
III & 185 & 3000 & 110 & $\mathbf{6 0}$ \\
IV & 185 & 3000 & 110 & $\mathbf{7 5}$ \\
V & 185 & 3000 & 110 & $\mathbf{9 0}$ \\
\hline
\end{tabular}

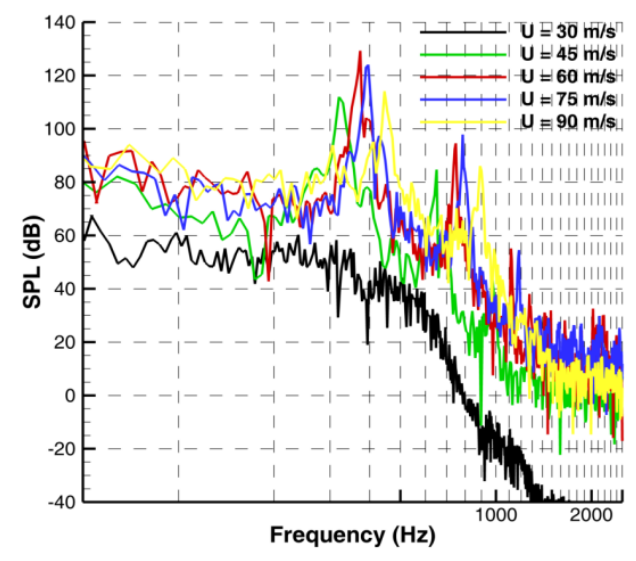

a)

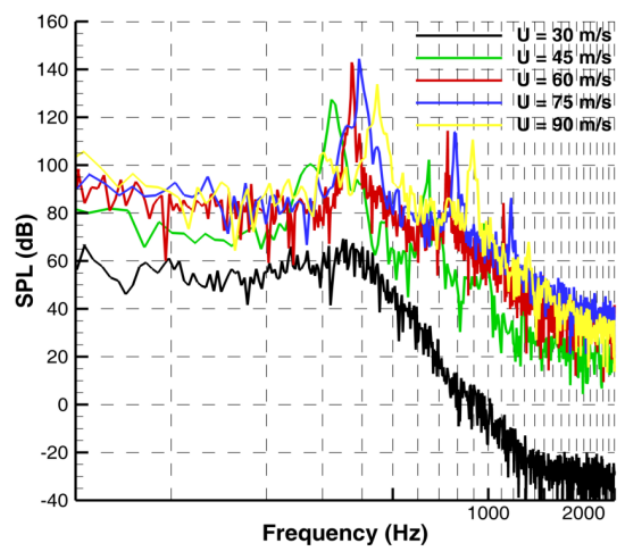

b)

Fig. 10 Sound pressure frequency spectrum curve: a) p2; b) p5 In Fig.10 it can be seen that under the condition of low 
velocity $(30 \mathrm{~m} / \mathrm{s})$, the resonant noise could not be excited either in the main pipe or in the stub pipe.

With the increase of the flow rate, the level of the noise begins to increase first, and in certain speed range $(60 \mathrm{~m} / \mathrm{s} \sim$ $75 \mathrm{~m} / \mathrm{s}$ ) it can reach the maximum value. However, with the continuous increase of the flow rate, the noise then intensity decreases after the peak value. In the calculation of the speed range, the noise shows monotonic frequency characteristic, the peak frequency moves to the high frequency band with the increase of flow rate, which is basically the same as the change of modal frequency characteristics. The peak frequencies were $325 \mathrm{~Hz}(45 \mathrm{~m} / \mathrm{s}), 375 \mathrm{~Hz}(60 \mathrm{~m} / \mathrm{s}), 395 \mathrm{~Hz}$ $(75 \mathrm{~m} / \mathrm{s})$ and $450 \mathrm{~Hz}(90 \mathrm{~m} / \mathrm{s})$ respectively.

The curve of SPL changed with flow rate at P2 and P5 are shown in Fig. 11. Low velocity $(30 \mathrm{~m} / \mathrm{s}$ ) fails to excite the resonant noise, and the SPL at the measured point P2 is almost the same as the measuring point P5. While the SPL at the measuring point P5 is larger than that at the measuring point P2 under other flow velocity. Total SPL with the velocity variation is not linear, it first increases with the speed, and when it reaches its maximum value, it decreases gradually with the increasing speed.

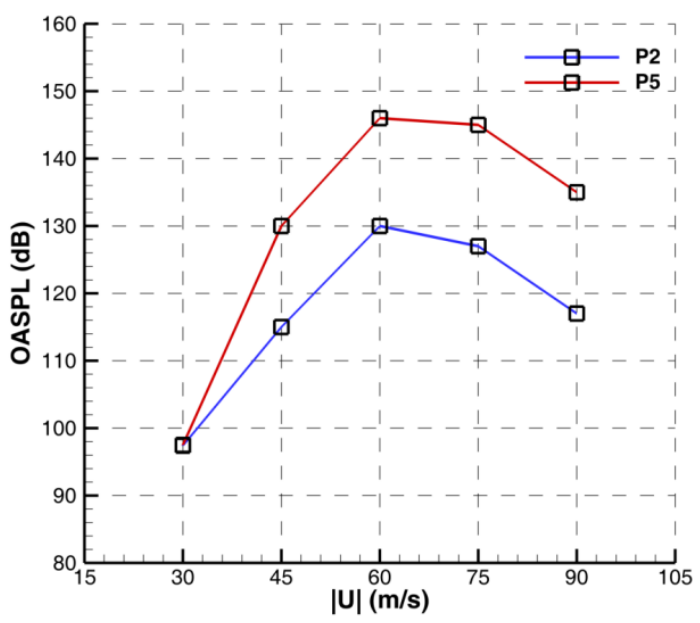

Fig. 11 the total SPL changed with velocity

\section{THEORETICAL ANALYSIS}

In this part the theoretical model and numerical calculation results were combined to analyze acoustic noise propagation.

\subsection{MATHEMATICAL MODEL OF NOISE PROPAGATION}

Based on the numerical simulation results, combined with the theory of sound propagation, it can be formed the following mathematical model.

There is one assumption that the accoustic wave propagated in the form of plane wave.

Based on the general complex solution of wave equation, the general form of the incident wave and the reflected wave can be expressed as:

Incident wave:

$$
p_{i}=p_{i a} e^{j(\omega t-k x)}
$$

Reflected wave:

$$
p_{r}=p_{r a} e^{j(\omega t+k x+\varphi)}
$$

Where $\mathrm{p}$ is Amplitude, $\varphi$ is the phase difference produced by reflection.

The sound pressure reflection coefficient as the ratio of the reflected wave to the incident wave can be defined by:

$$
r_{p}=\left.\frac{p_{r}}{p_{i}}\right|_{x=0}=\frac{p_{r a}}{p_{i a}} e^{j \varphi}
$$

So, the amplitude ratio before and after reflection can be used to define the acoustic pressure amplitude reflection coefficient:

$$
\left|\mathrm{r}_{\mathrm{p}}\right|=\frac{\mathrm{p}_{\mathrm{ra}}}{\mathrm{p}_{\mathrm{ia}}}
$$

The total sound pressure can be expressed as:

$$
\begin{aligned}
p_{a}=p_{i}+p_{r}= & p_{i a} e^{j(\omega t-k x)}(1 \\
& \left.+\left|r_{p}\right| e^{j(2 k x+\varphi)}\right)
\end{aligned}
$$

The amplitude is:

$$
\begin{aligned}
& \left|p_{a}\right|=p_{i a} \sqrt{1+\left|r_{p}\right|^{2}+2\left|r_{p}\right| \cos (2 k x+\varphi)} \\
& \text { if } 2 k x+\varphi= \pm 2 n \pi,\left|p_{a}\right|_{\max }=p_{i a}+p_{r a} \\
& \text { if } 2 k x+\varphi= \pm(2 n+1) \pi,\left|p_{a}\right|_{\text {min }}=p_{i a}-p_{r a}
\end{aligned}
$$

Therefore, the acoustic wave is the linear superposition of the incident wave and the reflected wave, and the standing wave effect will occur.

\subsection{MODEL NUMERICAL VERIFICATION}

Fig.12 Fig.14 show the distribution of SPL under different length of the main pipe (3000 mm vs. $2300 \mathrm{~mm}$ ) and different velocity of main pipe (45 m/s vs. $60 \mathrm{~m} / \mathrm{s}$ ). The right graphs in Fig.12 Fig.14 show the distribution of SPL with the length of the main pipe. It can be found that there was standing wave effect on upstream direction.

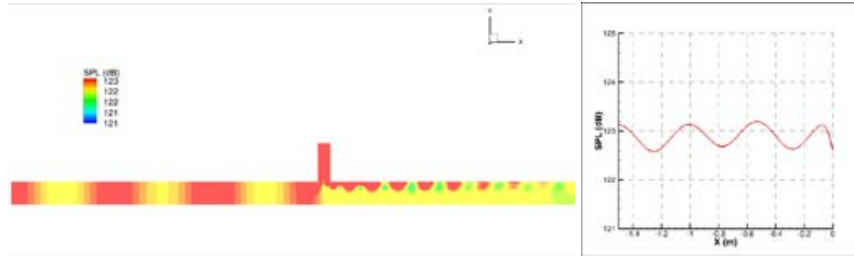

Fig.12 Distribution of SPL $(\mathrm{L}=3000 \mathrm{~mm}, \mathrm{~V}=45 \mathrm{~m} / \mathrm{s})$

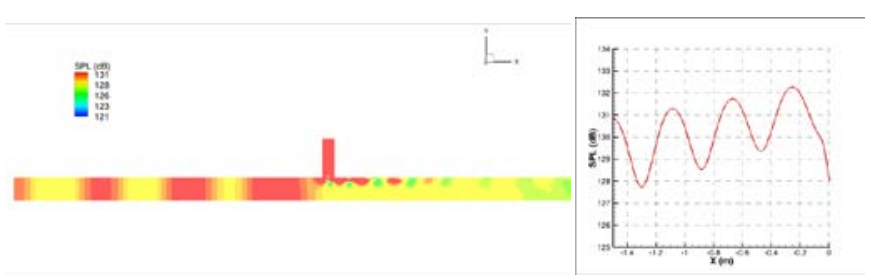

Fig.13 Distribution of SPL $(\mathrm{L}=3000 \mathrm{~mm}, \mathrm{~V}=60 \mathrm{~m} / \mathrm{s})$

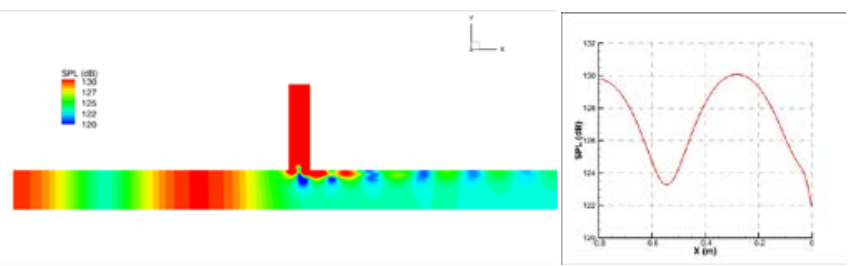

Fig.14 Distribution of SPL $(\mathrm{L}=2300 \mathrm{~mm}, \mathrm{~V}=60 \mathrm{~m} / \mathrm{s})$ 
Based on the mathematical model, the wavelength of standing wave can be calculated, and the result can be compared with numerical result. Using the formula (6), when the peak wavelength was satisfied, the following formula was established:

$$
2 \mathrm{k} \Delta \mathrm{x}=2 \pi, \mathrm{k}=2 \pi / \lambda
$$

So the wavelength of standing wave is:

$$
\Delta \mathrm{x}=\lambda / 2
$$

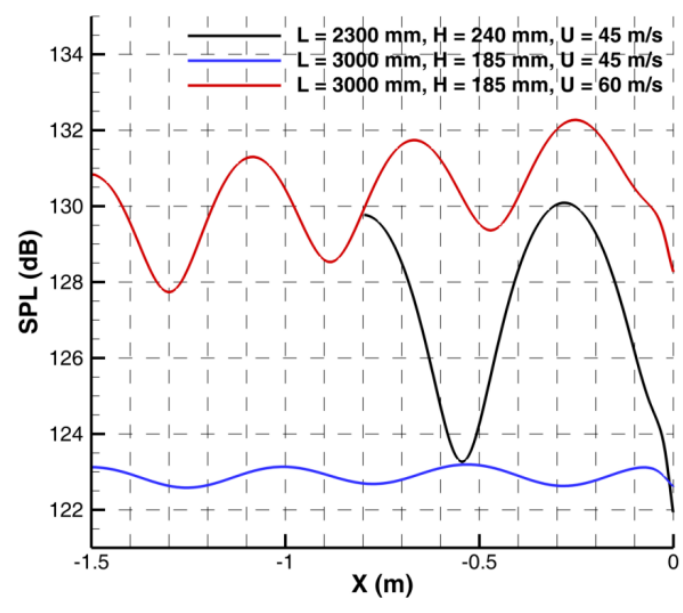

Fig. 15 the distribution of SPL of upstream direction

Fig.15 compares the distribution of SPL of upstream direction under different flow velocities and different length of the pipe.

When the flow velocity $(45 \mathrm{~m} / \mathrm{s})$ is the same, it can be found that the wavelength of standing wave (blue line and black line) is the same, the wavelength value is approximately $0.52 \mathrm{~m}$. According to the numerical simulation results of Section 2.4, the main peak frequency under this velocity is about $325 \mathrm{~Hz}$. Therefore, the wavelength under the frequency is:

$$
\lambda=\frac{340}{325}=1.05 \mathrm{~m}
$$

Using equation (8) it can be solved that the wavelength of standing wave under the frequency is $0.525 \mathrm{~m}$ which is almost the same as the numerical result.

When the flow velocity is $60 \mathrm{~m} / \mathrm{s}$, the main peak frequency was obtained about $375 \mathrm{~Hz}$ by the numerical simulation. Using formula (8) it can be obtained that the wavelength of standing wave by theoretical model is $0.453 \mathrm{~m}$ which is almost the same as the numerical result $0.432 \mathrm{~m}$.

Based on the theoretical model, it can be found that the spread of noise in the upstream of the main pipe has the following rules: the anterior length of the main pipe does not affect the maximum SPL, but due to the presence of standing wave effect, it affected the distribution of sound pressure in the upstream of the main pipe.

\section{CONCLUSIONS}

This paper combined with theoretical modeling and numerical simulation analysis to investigate the generation mechanism and propagation characteristics of aerodynamic noise. The main conclusions were as follows:

- The effect of the height of the stub pipe on the peak frequency is not obvious, but SPL increases as the height of stub pipe increases.

- The noise with $110 \mathrm{~mm}$ diameter appears obvious monotonic peak frequencies, while the noise of $220 \mathrm{~mm}$ diameter is more like a broadband noise.

- The effect of the length changes of the main pipe on SPL can be ignored.

- The total SPL with the velocity variation is not linear. It first increases with the increasing speed, and when it reaches the maximum value, it decreases gradually with the increasing speed.

- The interaction between the air flow of the main pipe and the stub pipe will produce obvious monotonic frequency noise. The noise is propagated upstream in the form of plane wave which makes the standing wave effect. the anterior length of the main pipe does not affect the maximum SPL, but due to the presence of standing wave effect, it affected the distribution of sound pressure in the upstream of the main pipe

\section{ACKNOWLEDGMENTS}

This work is supported by the fund of Multipurpose Engineering and General Application Platform in China (No. KW064-ID-2015).

\section{REFERENCES}

[1].Daniel V. Summerville. Scaling laws for model test based BWR steam dryer fluctuating load definitions [C]. 2006 ASME PVP , ICPVT-11-93703.

[2].Masaya Ohtsuka, Kiyoshi Fujimoto, et al. Study on acoustic resonance and its damping of BWR steam dome [C]. 2006 ICAPP, 6186.

[3].Shiro Takahashi, Masaya Ohtsuka, et al. Experimental study of acoustic and flow-induced vibrations in BWR main steam lines and steam dryers [C]. 2008 ASME PVP, 61318.

[4].Keita Okuyama, Shiro Takahashi, et al. Flow visualization of acoustic resonance for safety relief valves in power up-rated BWRs [C]. 2009 ICONE 17-75035.

[5].Samir Ziada. Flow-excited acoustic resonance in industry [C]. 2010 ASME JOPVT-015001-1.

[6].Ziada, S. and Shine, S. Strouhal Numbers of Flow-Excited Acoustic Resonance of Closed Side Branches. Journal of Fluids and Structures, 1999, Vol. 13, pp. 127-142.

[7].Hambric, S.A., Mulcahy, T.M., Shah, V.N., et. al. Flow-Induced Vibration Effects on Nuclear Power Plant Components Due to Main Steam Line Valve Singing. 2009 Proceedings of the Ninth NRC/ASME Symposium on Valves, Pumps and Inservice Testing, NUREG/CP-0152, Vol. 6, pp. 3B:49-3B:69.

[8].Stoneman, S. A. T., Hourigan, K., Stokes, A. N., and Welsh, M. C., 1988,"Resonant Sound Caused by Flow Past Two Plates in Tandem in a Duct,” J. Fluid Mech., 192, pp. 455-484

[9].Hourigan, K., Welsh, M. C., Thompson, M. C., et. al. Areodynamic Sources of Acoustic Resonance in a Duct With Baffles. 1990, J. Fluids Struct., 4, pp. 345-370.

[10].Hall, J. W., Ziada, S., and Weaver, D. S., The Effect of Applied Sound on Vortex Shedding From Two Tandem Cylinders, 2003, J. Fluids Struct., 18, pp. 741-758. 